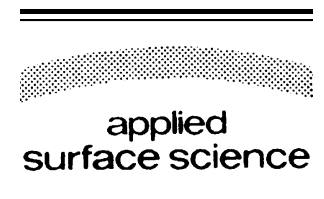

Applied Surface Science 249 (2005) 12-15

www.elsevier.com/locate/apsusc

Short communication

\title{
Enhanced peak separation in XPS with external biasing
}

\author{
Gulay Ertas, U. Korcan Demirok, Sefik Suzer* \\ Bilkent University, Chemistry Department, Ankara 06800, Turkey
}

Received 23 October 2004; received in revised form 29 November 2004; accepted 30 November 2004

Available online 6 January 2005

\begin{abstract}
We have demonstrated that the $\mathrm{Au} 4 \mathrm{f}$ peaks of the capped gold nanoparticles deposited on a $\mathrm{SiO}_{2}(20 \mathrm{~nm}) / \mathrm{Si}$ substrate can be separated form the $\mathrm{Au} 4 \mathrm{f}$ peaks of a gold metal strip, in contact with the same sample, by application of an external voltage bias to the sample rod while recording the XPS spectra. The external bias controls the flow of low-energy electrons falling on to the sample which in-turn controls the extent of the differential charging of the oxide layer leading to shifts in the binding energy of the gold nanoparticles in contact with the layer. The method is simple and effective for enhancing peak separation and identification of hetero-structures.
\end{abstract}

(C) 2004 Elsevier B.V. All rights reserved.

Keywords: External biasing; Differential charging; Peak separation; Gold nanoclusters

$\mathrm{X}$-ray photoelectron spectroscopy (XPS) is the leading analytical technique for characterizing various chemical/physical forms of elements in surface structures. The characterization is solely based on associating the changes in measured binding energies of certain atomic levels with chemical or physical forms of the corresponding elements [1]. It is also well-established that the measured binding energies are affected by numerous internal and external parameters, like size, morphology, and thermal history of the sample, nature of the surrounding medium, methods used for charge neutralization, etc. [2-9]. In

\footnotetext{
* Corresponding author. Tel.: +90 3122664946; fax: +903122664579 .

E-mail address: suzer@fen.bilkent.edu.tr (S. Suzer).
}

addition, as we had recently shown, application of a small $(0-10 \mathrm{~V})$ external bias to the sample rod while recording the XPS spectrum, also influences the measured binding energies [10]. The external bias applied controls the flux of slow electrons (stray electrons and/or electrons from a filament) falling onto the sample which normally neutralize the positive charge developed as a result of the photoemission process [11]. It was also demonstrated that this control of charging can be utilized to separate some of the otherwise overlapping spectral features or extracting proximity information about surface structures [12]. Discussion about charging (especially differential charging) is not new and goes back more than three decades [13-16], but only recently several applications have appeared in the literature which utilize it for 
extracting useful chemical, physical and electrical information about the systems investigated [17-22]. In this letter, we present one simple application of the differential charging, whereby controlling it, leads to total spectral separation of the XPS peaks of gold in two different physical forms (metallic gold and gold nanoclusters) present on the same surface.

Differentiation on the basis of chemical shifts of the different chemical forms of gold in the $\mathrm{Au}^{3+}, \mathrm{Au}^{1+}$, and $\mathrm{Au}^{0}$ is routine in XPS since the corresponding binding energies are significantly different from each other (for $\mathrm{Au} 4 \mathrm{f}_{7 / 2}$ they are 86.7, 85.2, and $84.00 \mathrm{eV}$, respectively $[1,23,24])$. Secondly, the size of the clusters also influences the binding energy of the atom, size-dependent shift, the origin of which is still actively debated [6-9]. In this respect, Luo et al. recently reported that the binding energy of small nanoclusters of gold, prepared by physical vapor deposition, can be as much as $1.8 \mathrm{eV}$ higher $(85.8 \mathrm{eV})$ compared to that of the metallic gold [6]. Furthermore, there are also reports indicating that some measurable binding energy differences exist with respect to the matrix where the gold clusters are residing [7-9]. Experimentally, it is straightforward to measure and quantify the contribution of each parameter separately (i.e. one at a time), but severe difficulties arise when two or more of these coexist and their contributions complicate the spectrum due mostly to insufficient instrumental resolution. In certain cases, deconvolution techniques are of help, but it is obvious that other experimental tools/tricks are also welcomed.

Gold nanoparticles capped with tetraoctylammonium bromide (TOAB) in toluene were prepared using the well-established routes [25]. The resulting solution displays a red-wine color and has a strong absorption peak around $530 \mathrm{~nm}$ as determined by the UV-vis spectrometer [26,27]. When the gold nanoparticles aggregate the color of the solution becomes black and the absorption band shifts to longer wavelengths.

A drop of the solution containing the gold nanoclusters was deposited onto silicon substrates with various thermal oxide thicknesses $(2-40 \mathrm{~nm})$. The sample is tied onto the XPS sample holder together with a gold metal strip. A KRATOS ES300 spectrometer with non-monochromatic $\mathrm{Mg} \mathrm{K} \alpha \mathrm{X}$-ray source is used to record the XPS data. External bias is applied to the sample rod while recording the spectra and a filament is placed ca. $5 \mathrm{~cm}$ away from the sample to provide additional low-energy electrons. Fig. 1 depicts the set-up as well as the Si $2 p-A u 4 f$ region of the XPS spectra recorded with and without external bias. An ca. $20 \mathrm{~nm} \mathrm{SiO} / 2 / \mathrm{Si}$ substrate is chosen so that only the $\mathrm{Si} 2 \mathrm{p}$ of the oxide is accessible by XPS. This choice is dictated for reasons of spectral simplicity and clarity, since for samples thinner than $10 \mathrm{~nm}$, an additional Si 2p peak of the silicon metal around $100 \mathrm{eV}$ would also be present. The inset shows a picture of the solution of the red-wine gold nanoclusters.

As mentioned above, our method is based on the ability to measure the local potential developed as a result of the different response of the gold metal and the $20 \mathrm{~nm} \mathrm{SiO}{ }_{2}$ dielectric layer (on which the gold nanoparticles reside) to different electron fluxes guided by the external bias applied. As shown in Fig. 1(b), two peaks of the Au 4f spin-orbit doublet belonging to the nanoclusters and the bulk gold overlap when the sample is grounded which is the normal practice employed. When the sample is biased at $-10 \mathrm{~V}$ the Si $2 \mathrm{p}$ of the oxide shifts to higher binding energy due to the enhanced differential (positive) charging, and the gold peaks separate into two doublets as shown in Fig. 1(c). One of the doublets does not shift but the other shifts almost as much as the Si 2 p peak. With application of $+10 \mathrm{~V}$ the silicon peak shifts in the opposite direction and becomes broader, and one of the gold doublets follows it as given in Fig. 1(a). In this case the oxide is overneutralized by the electrons from the filament, and has an overall negative potential accompanied with an increase in FWHM, which is also reflected in the Au $4 \mathrm{f}$ of the nanoclusters.

An oversimplified schematics of the different process is depicted in Fig. 2. In the negative bias region there is a net loss of electrons through the photoemission process into the vacuum, and assuming the silicon oxide layer to have a finite resistance, a voltage drop, equal to IR, develops. This in-turn increases the measured binding energy difference between the gold metal and the silicon oxide layer. As the bias is made positive low-energy stray electrons and electrons from the filament compensate the loss of electrons to the extent that a net electron flow to the sample results. In this case, the IR drop now changes its sign and causes the measured binding energy difference to decrease. Since the gold metal strip is 

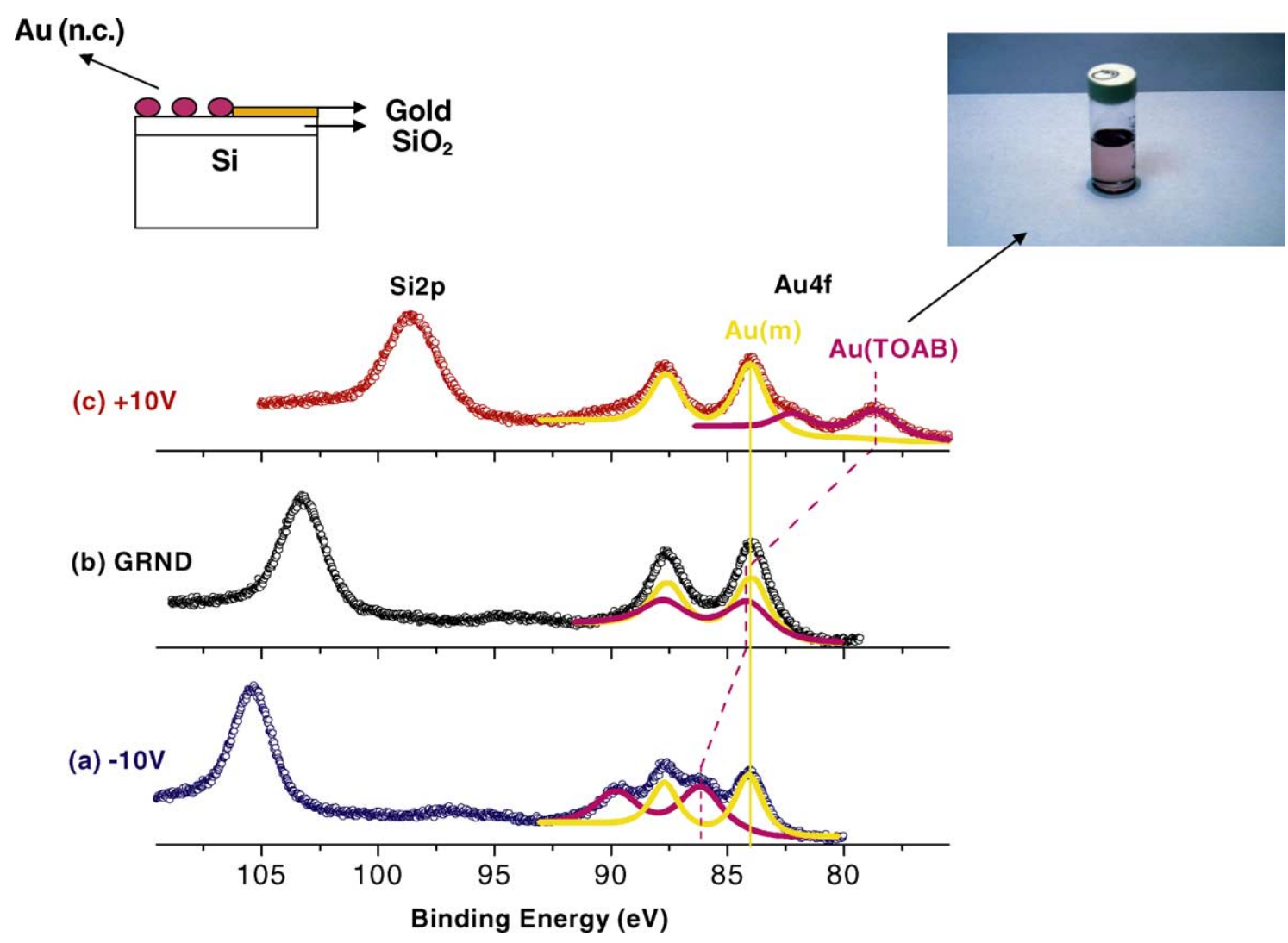

Fig. 1. Si 2p-Au $4 \mathrm{f}$ region of the XPS spectrum of a ca. $20 \mathrm{~nm} \mathrm{SiO} / 2 / \mathrm{Si}$ substrate onto which one drop a solution containing tetraoctylammonium bromide is deposited: (b) without, (a) with $-10 \mathrm{~V}$, and (c) with $+10 \mathrm{~V}$ external bias applied to the sample rod. The inset on the top left depicts the experimental set-up, and the one on top right depicts a picture of the solution of the gold nanoclusters.

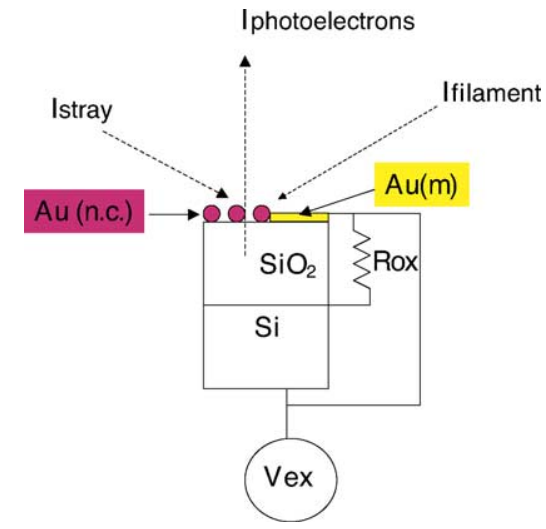

Fig. 2. A simplified schematics of the various electron processes and electrical connections. tied to the sample rod it follows the external potential applied, but since the gold nanoparticles are in electrical contact with the oxide layer they more or less follow the same voltage path as the silicon oxide layer. There are also small variations (up to about $0.5 \mathrm{eV}$ ) in the measured binding energy difference between the peak belonging to the gold nanoclusters and that of the oxide. This could be attributed to parameters, like presence of a finite impedance between the nanoclusters and the oxide layer, and/ or size-dependent shift in the binding energy, etc. Understanding and quantification of these parameters are also very important but fall beyond the scope of this letter since extensive theoretical and experimental investigations are needed for elucidating them.

In conclusion we have demonstrated that by imposing a simple external voltage stress to the sample rod, while acquiring XPS data, we have been 
able to control and measure the potential(s) developed as a result of charging in and on different layers/ domains. This simple procedure has been turned into a powerful tool for enhancing peak separation. The method is general and versatile for extracting additional chemical (qualitative and quantitative), physical and (non-contact) electrical information about various surface structures.

\section{Acknowledgements}

This work was supported by TUBA (Turkish Academy of Sciences), and by TUBITAK (The Scientific and Technical Research Council of Turkey) through Grant No. TBAG-2261.

\section{References}

[1] D. Briggs, M.P. Seah, Practical Surface Analysis, Wiley, Chichester, 1996.

[2] G.K. Wertheim, S.B. Dicenzo, S.E. Youngquist, Phys. Rev. Lett. 5 (1983) 2310-2313.

[3] M.G. Mason, Phys. Rev. B 27 (1983) 732-742.

[4] S. Iwata, A. Ishizaka, J. Appl. Phys. 79 (1996) 6653-6713.

[5] H. Kobayashi, T. Kubota, H. Kawa, Y. Nakato, M. Nishiyama, Appl. Phys. Lett. 73 (1998) 933-935.

[6] K. Luo, D.Y. Kim, D.W. Goodman, J. Mol. Catal. A: Chem. 167 (2001) 191-198.

[7] T. Ohgi, H.Y. Sheng, Z.C. Dong, H. Nejoh, D. Fujita, Appl. Phys. Lett. 79 (2001) 2453-2455.
[8] T. Ohgi, D. Fujita, Phys. Rev. B 66 (2002) 115410-115415.

[9] A. Howard, D.N.S. Clark, C.E.J. Mitchell, R.G. Egdell, V.R. Dhanak, Surf. Sci. 518 (2002) 210-224.

[10] B. Ulgut, S. Suzer, J. Phys. Chem. B 107 (2003) 2939-2943.

[11] F. Karadas, G. Ertas, S. Suzer, J. Phys. Chem. B 108 (2004) 1515-1519.

[12] S. Suzer, Anal. Chem. 75 (2003) 7026-7029.

[13] T. Dickinson, A.F. Povey, P.M.A. Sherwood, J. Electron. Spectrosc. Rel. Phenom. 2 (1973) 441-447.

[14] T.L. Barr, J. Vac. Sci. Technol. A 7 (1989) 1677-1683.

[15] A. Cros, J. Electron. Spectrosc. Rel. Phenom. 59 (1992) $1-14$.

[16] J. Cazaux, J. Electron. Spectrosc. Rel. Phenom. 105 (1999) $155-185$.

[17] J.H. Thomas, C.E. Bryson, T.R. Rampalone, J. Vac. Sci. Technol. B 6 (1988) 1081-1086.

[18] W.M. Lau, Appl. Phys. Lett. 54 (1989) 338-340.

[19] H. Kobayashi, K. Namba, Y. Yamashita, Y. Nakato, T. Komeda, Y. Nishioka, J. Appl. Phys. 80 (1996) 1578-1582.

[20] N.J. Havercroft, P.M.A. Sherwood, Surf. Interface Anal. 29 (2000) 232-240.

[21] H. Doron-Mor, A. Hatzor, A. Vaskevich, T. Van der BoomMoav, A. Shanzer, I. Rubinstein, H. Cohen, Nature 406 (2000) 382-385.

[22] H. Cohen, Appl. Phys. Lett. 85 (2004) 1271-1273.

[23] G. Battistoni, G. Mattogno, R. Zanoni, L. Naldini, J. Electron. Spectrosc. Rel. Phenom. 28 (1982) 23-32.

[24] C. Battistoni, G. Mattogno, D.M.P. Mingos, J. Electron. Spectrosc. Rel. Phenom. 33 (1984) 107-114.

[25] M. Brust, M. Walker, D. Bethell, D.J. Schiffrin, R. Whyman, J. Chem. Soc., Chem. Commun. (1994) 801-802.

[26] M.M. Alvarez, J.T. Khoury, T.G. Schaaff, M.N. Shafigullin, I. Vezmar, R.L. Whetten, J. Phys. Chem. B 101 (1997) 37063712.

[27] A.N. Shipway, M. Lahav, R. Gabai, I. Willner, Langmuir 16 (2000) 8789-8795. 